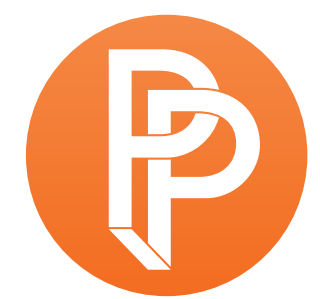

PERFORMANCE

PHILOSOPHY

\title{
NIETZSCHE EIN IMMANENTIST? ${ }^{1}$
}

\section{ANDREASURS SOMMER UNIVERSITA'T FREIBURG}

Transkription des deutschen gesprochenen Textes: Eva-Maria Aigner

Good morning, guten Morgen, meine Damen und Herren!

This morning's lecture will be a rather unusual performance. First, you will be able to enjoy a very old-fashioned kind of lecture, as I won't present any PowerPoint slides; and second, I will be presenting my paper primarily in German. Since this could be problematic for some, I will provide English abstracts summarizing the main points. I will be using the German for two reasons: It seems rather unusual to present an author and his philosophy in a foreign language and not the language in which he lived when the greater part of the audience shares the author's language. In Paris, nobody would deliver a lecture on Descartes or Sartre in English or Swahili to a mainly French audience; and in London, no one would ever present a paper on John Stuart Mill or Thomas Hobbes in German or Swahili. The main reason I am refraining from the use of English is not the quite snobbish pretention of protecting my own language. Rather, it is the fact that it is not possible for me to show you the nuances of Nietzsche's work in English; Nietzsche is a master of nuances. In the third portion of my paper, you will see that the English translations in our conference reader are terribly misleading in at least two cases; when we try to interpret Nietzsche, we should refer back to the original language, not only in reading him but also in presenting him-at least I, for one, feel obliged to do so. 
My paper consists of three chapters. The first chapter deals with Nietzsche's usage of the concept of "immanence;" I will be tracing his usage of this term. My second point is a more general one. I would like to focus on the question as to whether it is possible for Nietzsche to have a strong concept of immanence, particularly when we recall that Nietzsche clearly formulates his strong opposition to all ideas of transcendence. Might any immanence at all be retained here? The third chapter contains an in-depth examination of two sections of Beyond Good and Evil, Sections 3 and 6. I think we are able to note some important aspects in these passages as we seek to understand whether Nietzsche has any kind of idea or concept of immanence.

Ich komme damit zum ersten Punkt und zu den Stellen, die sich in Nietzsches Werk mit "Immanenz" und „immanent" beschäftigen. Wir müssen uns in diesem Zusammenhang vergegenwärtigen, dass der Immanenzbegriff, wie wir inn jetzt an dieser Tagung benutzen, als Oppositionsbegriff zur Transzendenz philosophiehistorisch eine relativ junge Erfindung ist. Für den Autor, der im Hintergrund all unserer Erörterungen steht, für Baruch de Spinoza, ist der Gegenbegriff zu „immanent" nicht „transzendent", sondern „transient", also „vorübergehend“. Immanenz steht Transienz gegenüber. Die Substanz, die in den Modi immanent ist, ist eben keine vorübergehende Substanz. Diese auf die Scholastik zurückgehende Begriffsverwendung hat Ende des 18. Jahrhunderts eine fundamentale Umstellung erfahren in Kants Kritik der reinen Vernunft, wo wir der uns gewohnten Unterscheidung immanent/transzendent erstmals begegnen. Ich will das nicht im Einzelnen ausführen, sondern es nur als Hintergrundinformation vorausschicken: eine Hintergrundinformation, die wichtig ist, wenn wir uns die Verwendung von Worten aus dem Begriffsfeld "Immanenz" vergegenwärtigen. Es sind, wenn man es sich dann anschaut, nicht viele Stellen, die man bei Nietzsche zu Immanenz findet. Es wären hingegen sehr viel mehr Stellen, die man zu Transzendenz fände, die will ich aber nicht mit Ihnen besprechen. Es sind im Übrigen Stellen, die großteils aus dem Nachlass stammen-wenn ich es richtig überblicke, gibt es nur zwei einschlägige Stellen in den von Nietzsche publizierten Werken (Menschliches, Allzumenschliches I, Abschnitt 96 und Die fröhliche Wissenschaft, Abschnitt 292). Bei Nietzsche neigt man dazu, den Nachlass überzubewerten; das ist eine alte Sucht der Nietzsche-Forschung, nämlich so zu tun, als sei das Wichtige im Nachlass versteckt und das Eigentliche dort zu finden. Richtig hingegen ist: Nachlass ist in erster Linie Werkstatt, Laboratorium und nicht fertiges Produkt. Nietzsche war sehr wohl im Stande, das eine vom anderen zu unterscheiden, was leider heutige Interpreten häufig nicht sind, indem sie dann irgendeine scheinbar passende Stelle aus dem Nachlass herausklauben und zum Angelpunkt ihrer Nietzsche-Deutung machen. Das heißt nicht, dass man den Nachlass ganz ausklammern sollte. Um begriffliche Entscheidungen in Nietzsches Werken zu verstehen, ist es durchaus hilfreich, den Nachlass als Reservoir heranzuziehen.

Die chronologisch erste Stelle, die sich mit Immanenz beschäftigt, ist eine aus dem Nachlass von 1869, die von der Religion der Griechen handelt. Sie bereitet die Konzeption der griechischen Religion in der Geburt der Tragödie vor, und da heißt es: „Die Religion für das Leben: ganz immanent: Religion der Schönheit als der Blüthe, nicht des Mangels. Weder Pessimisten noch Optimisten." (NF-1869, 3[42])—Noch einmal: „Die Religion für das Leben: ganz immanent”. Sie sehen, meine 
Damen und Herren, die Grundopposition, die Sie sich dazu denken müssen, die Grundopposition zum Christentum, das den Lebensmittelpunkt auf das Jenseits hin verlagert. Also eine sehr frühe Parteinahme, wenn Sie so wollen, für Immanenz, aber noch nicht im eigentlichen Sinne programmatisch, sondern eine religionswissenschaftliche Feststellung, die Nietzsche im Übrigen in der von ihm gelesenen Literatur auch vielfach hatte finden können.

Was ihn dann, wenn man die Nachlassstellen weiter durchgeht, interessiert, ist die Frage, ob es immanente Gesetze und Maße im Universum gibt. Die also nicht von außen aufgedrückt sind, von Göttern beispielsweise, sondern die sich aus der inneren Sachlogik des Weltverlaufes ergeben. Das sind Vorstellungen, die Nietzsche insbesondere in der vorsokratischen Philosophie findet. Er nimmt Überlegungen von Empedokles und Heraklit auf, die immanente Gesetzmäßigkeiten im Weltganzen zu postulieren scheinen. Von den im "Kampfe immanenten Gesetzen und Maaßen“ spricht die nachgelassene, von Nietzsche nicht veröffentlichte Abhandlung Die Philosophie im tragischen Zeitalter der Griechen im 5. Abschnitt; während es, auf Heraklit gemünzt, im 7. Abschnitt heißt: „In dem höchsten und in dem verkehrtesten Menschen offenbart sich die gleiche immanente Gesetzmäßigkeit und Gerechtigkeit." (PHG-7)

Nun könnte man denken, Nietzsche sei fasziniert von dieser Vorstellung einer immanenten Ordnung, einer immanenten Form der Gerechtigkeit. Wenn man sich dann aber weiter umschaut, in Nietzsches Nachlass insbesondere-wie gesagt, die Werke geben wenig her, da scheint er den Immanenzbegriff nicht wirklich für verwendungsfähig gehalten zu haben-wenn man sich also im Nachlass weiter umschaut, findet man Passagen wie die folgende: „Alle möglichen solchen Vorstellungen über ,immanente Gerechtigkeit', ,Heilsordnung', ausgleichende ,transcendente Gerechtigkeit' gehen jetzt in jedem Kopfe herum — sie bilden das Chaos der modernen Seele mit." (NF-1884, 26[279]) Man hätte von den vorhin zitierten frühen Äußerungen her die Vermutung haben können, Nietzsche spiele jetzt die Immanenz gegen die Transzendenz aus, er wolle immanente Gerechtigkeit gegen transzendente Gerechtigkeit in die Waagschale werfen. Nimmt man nun diese jüngere Passage, sind ganz offensichtlich Fragezeichen angebracht. Wenn man sich solche Nachlassstellen anschaut (es gibt noch weitere, z. B. NF-1885, 34[5], NF-1885, 40[662] und NF-1886, 6[9]), fallen die ausgesprochen kritisch aus. Sie implizieren, dass ein Reden von immanenter Gerechtigkeit im Anschluss an die Vorsokratiker genauso unter den Metaphysikverdacht fällt oder genauso eine unstatthafte Projektion zu sein scheint wie die Alternative, nämlich eine überirdische Heilsordnung, transzendente Gerechtigkeit. Es ist also nicht so, dass Nietzsche in den 1880er Jahren, nach Ausweis seines Nachlasses, eine Option für Immanenz gegen Transzendenz in dieser Begrifflichkeit geltend machen würde. Vielmehr entsteht der Eindruck, dass Immanenz-Vorstellungen, die harmonistische, ontologisch beschönigende Vorstellungen sind, genauso der Kritik verfallen müssen wie diejenigen, die besagen, es gebe eine transzendente Ordnung, die uns übergestülpt ist. Mit dem Immanenzbegriff selber wird man schwerlich operieren können, wenn man versucht, Nietzsche in seiner ideengeschichtlichen Oppositions-Position klar zu profilieren. Die einzige Stelle, die tatsächlich für Immanenz als Begriff, als Wort in Nietzsches Werk (und ich spreche jetzt nicht mehr vom Nachlass, sondern von den Werken) relevant ist, ist Fröhliche Wissenschaft, Abschnitt 292, und da ist "von der Gerechtigkeit und der immanenten Vergeltung" die Rede, die manche „an die Spitze [ihrer] Moral“ stellen würden 
und „von früh bis Abend" davon reden. Eine „Popularität und ein Geschrei der Gasse" sei das, so das Resümee. Also erscheint die Vorstellung der immanenten Vergeltung, dass die Dinge sich sozusagen ins Lot bringen auf Grund einer inneren Ordnung, die irgendwie da ist und im Weltganzen gründet, dem sprechenden Ich in Fröhliche Wissenschaft, Abschnitt 292 als eine lachhafte metaphysische Annahme. Das ist ganz auf der Linie dessen, was wir im Nachlass der 1880er Jahre zum Immanenzbegriff finden. Wir müssen hier in Erinnerung rufen, dass Immanenz als Gegenbegriff zu Transzendenz von vielen- Nietzsche wohlbekannten—religionskritischen Autoren während der zweiten Hälfe des 19. Jahrhunderts aufgerufen wurde (z. B. Ludwig Feuerbach, Max Stirner und Eduard von Hartmann). Diese Autoren versuchten, eine Immanenzphilosophie zu etablieren, um sich der Transzendenz-Bezogenheit zu entledigen. Nietzsche findet dieses Ansinnen ganz offensichtlich eher komisch oder hält es für ein "Geschrei der Gasse". Es ist eben Zeichen für "das Chaos der modernen Seele“, dass man so etwas annimmt oder womöglich alles zusammenmanscht, beispielsweise immanente und transzendente Gerechtigkeit: Das moderne Individuum hat offenbar die Tendenz, die Dinge heillos zu verwirren, wenn nur seine Trost- und Sinnbedürfnisse befriedigt werden.

Offensichtlich ist mit dem Begriff der Immanenz bei Nietzsche nicht viel anzufangen. Das muss jedoch nichts heißen gegen eine nicht an diesem Begriff festgemachte immanenzphilosophische Interpretation Nietzsches. Man könnte ja ohne Weiteres argumentieren, Nietzsche habe den Begriff der Immanenz vielleicht nicht gemocht und dagegen grundlegende Reserven angemeldet. Dies müsse jedoch nicht bedeuten, dass er eine Immanenzphilosophie, wie wir sie von Gilles Deleuze her kennen, prinzipiell hätte ablehnen müssen. Vielleicht bediente Nietzsche sich einfach nur einer anderen Begrifflichkeit.

A short abstract of this first part: I argue that in Nietzsche's posthumous fragments, he does not cast the concept of immanence in a very friendly light. Obviously, at the beginning of his thinking, he saw that a kind of immanent religion was possible, particularly in ancient Greece, and that the Presocratic philosophers had obviously believed in a kind of immanent order within the universe. On the other hand, Nietzsche is very critical in the 1880 s about the possibility of re-establishing an immanent order today; hence, the concept of "immanence" and the usage of this term seems unsuitable for contemporary thought and thought that could also be relevant for the future, since the term, according to Nietzsche, is still overloaded with metaphysical implications. Thus, freely making use of the term "immanence" and its underlying conceptual framework does not provide a means of escape when it comes to transcendence, for example, or the old notion of God. Something else has to be found. It might certainly be possible that one could translate criticism of the term "immanence" in Nietzsche into a philosophy of immanence-with the help of Deleuze, for instance. Perhaps Nietzsche is only critical of the term "immanence" but not of the issue behind the term. 
Ich komme damit zum zweiten Punkt. Die Marginalität des Immanenzbegriffes, die wir festgestellt haben, erstaunt nicht. Denn wenn man einen starken Begriff von Immanenz würde bilden wollen, bräuchte man einen Gegenbegriff. Und der Gegenbegriff, den uns die Philosophie seit Kant anbietet und der insbesondere in der hegelschen Tradition und auch in der anti-hegelschen Tradition bis zum Abwinken geltend gemacht worden ist, dieser Gegenbegriff ist der Begriff der Transzendenz, ich sagte es zu Beginn schon. Und dieser Denkweg ist für Nietzsche nicht mehr gangbar, da man bei der Immanenz immer nach dem Außen fragt—er ist nicht mehr gangbar, weil Nietzsche offensichtlich tatsächlich versucht, plurale Einheit zu denken. Er hat, könnte man deswegen sagen, Alternativen im Angebot für den Immanenzbegriff, der ihm kontaminiert erscheint. Die Hauptalternative ist der Begriff des Lebens, die Nebenalternative ist der Begriff des Willens zur Macht. Es handelt sich hier nicht um Lehren, um letzte Festlegungen Nietzsches. Es schiene mir viel angemessener, diese Überlegungen Nietzsches als intellektuelle Experimente zu charakterisieren. Nietzsche agiert nicht als Lehrer der ewigen Wiederkunft, sondern lässt gelegentlich einen persischen Propheten auftreten, der sich als Lehrer dieser Lehre gebärdet. Gleiches, oder Analoges, gilt auch vom Willen zur Macht oder vom Übermenschen. Man könnte sagen, Nietzsche vermeide den Begriff oder Worte aus dem Begriffsfeld der Immanenz deswegen, weil der Begriff nach einem "anders", nach einer Gegenoption schreit, und Nietzsche die nicht haben will. Man könnte ja sehr wohl argumentieren, Nietzsche sei ein eminenter Immanenzphilosoph, wenn er wiederum seine Prophetenfigur Zarathustra predigen lässt, man solle der Erde treu bleiben. Das klingt wie die Losung einer Immanenzphilosophie. Eine Losung, die eben auf die radikale Diesseitigkeit setzt. Bekanntermaßen wirft Nietzsche denjenigen, die noch befangen sind in Vorstellungen, es müsse einen Gott jenseits dieser Welt geben, vor, sie betrieben eine Schwergewichtsverlagerung hin zu einer diesseits-, welt- und lebensverneinenden Lebensform (und Lebensform muss man hier schon fast in Anführungszeichen setzen). Der Denunziationsgestus läuft bei Nietzsche darauf hinaus, dass diejenigen, die sich vom asketischen Ideal bestimmen lassen (vgl. dazu den Beitrag von Arno Böhler in diesem Band) diejenigen seien, die die radikale Immanenz nicht ernst nehmen wollen (wie gesagt, Nietzsche würde den Immanenzbegriff hier nie verwenden). Es sind diejenigen, die das Leben verneinen. Nietzsche als Verteidiger einer so verstandenen Immanenz hat also eine gewisse Plausibilität. Er ist der permanente Kritiker einer Verlagerung des Lebensschwergewichtes in ein Anderswo und in ein Anderswann.

Man hat bei Nietzsche auch Stellen, die ihn scheinbar als religiösen Denker ausweisen, und ich will nur auf eine Stelle hinweisen, weil ich es im Kommentar, den ich einmal zur Götzen-Dämmerung geschrieben habe (vgl. Sommer 2012), versäumt habe, die Stelle näher zu kommentieren,-ich hole das deswegen hier ganz kurz nach. Es geht um eine Passage in Götzen-Dämmerung oder Wie man mit dem Hammer philosophirt von 1888, eine Passage, in der das sprechende Ich sagt, es sei der "letzte Jünger des Philosophen Dionysos" (Götzen-Dämmerung, Was ich den Alten verdanke 5). Eine Feststellung, die ja scheinbar einen emphatischen Bekenntnischarakter hat. Es ist eine Stelle, die aufgegriffen wird in Ecce homo, Nietzsches Autogenealogie aus seinen letzten Schaffensmonaten. Da wird variiert und erweitert: „Ich bin ein Jünger des Philosophen Dionysos, ich zöge vor, eher noch ein Satyr zu sein als ein Heiliger." (Ecce homo, Vorwort 2) Das lässt einen schon aufhorchen, man fragt sich: ein positives Bekenntnis zu einer Art von Gegengott? 
Das Irritierende ist erstens: Über diese Dionysos-Figur erhält man in anderen Werken durchaus Auskunft, etwa in dem zwei Jahre vorher erschienenen Jenseits von Gut und Böse, Abschnitt 295. Dort wird Dionysos zunächst nicht als Philosoph eingeführt, sondern als Gott, aber nicht als ein normaler Gott, sondern als ein "Versucher-Gott". Ein Gott der höchsten Zweideutigkeit-dieser Dionysos scheint ein sehr merkwürdiger Gott zu sein. Nicht einfach, wie man sich das vorstellt, die Inkarnation der Lebensbejahung. Ein Versucher-Gott ist offensichtlich einer, der fundamental Gewissheiten in Frage stellt-zunächst wohl alle Gewissheiten, die man gemeinhin mit Göttern meint assoziieren zu können.

Zweitens: Merkwürdig in diesem Bekenntnis, der letzte Jünger des Philosophen Dionysos zu sein, ist das Bekenntnis zum Philosophen Dionysos. Seit wann ist Dionysos ein Philosoph, er ist doch ein Gott? Wenn überhaupt, würde man sagen, man bekenne sich zum Philosophen Jesus von Nazareth oder zum Philosophen Gautama Buddha? Eher nicht vermutlich, was will der Philosoph jetzt da? Wir haben ebenfalls in der Götzen-Dämmerung (Streifzüge eines Unzeitgemässen 19) Bruchstücke eines Zwiegesprächs von Dionysos mit Ariadne, Ariadne, die von Theseus verlassen worden ist und auf Naxos von Dionysos aufgegriffen wird. Sehr sonderbare Bruchstücke, die den Philosophen Dionysos zeigen, laut Nietzsche. Aber wer ist der Philosoph Dionysos im Unterschied zum Gott Dionysos? Können Götter philosophieren?

Dritter Punkt, auf den wir Acht geben sollten, wenn wir diese Passage auslegen: „ich bin der letzte Jünger des Philosophen Dionysos". Für diesen Satz gibt es mindestens zwei mögliche Interpretationen: Der letzte, weil keiner nachher kommt, weil die Bekenntnismöglichkeiten erschöpft sind. Andererseits, das ist die zweite Möglichkeit, wird man sich daran erinnern müssen, dass der letzte Jünger nach christlicher Terminologie Judas Ischariot ist, der Verräter. Derjenige, der die Auslieferung Jesu an die römischen Häscher ins Werk gesetzt hat, sich dafür bezahlen lässt mit den dreißig Silberlingen, sich danach aufhängt. Ist also der letzte Jünger des Philosophen Dionysos derjenige, der Dionysos verrät? Eine Interpretation, die vor dem Hintergrund einer Ankündigung Zarathustras noch eine gewisse Schärfe erhält, nämlich der Ankündigung an seine Jünger: „erst, wenn ihr mich Alle verleugnet habt, will ich euch wiederkehren“ (Also sprach Zarathustra I, Von der schenkenden Tugend, 3). Treue Jünger will Zarathustra auf gar keinen Fall haben.

Ich lasse das so dahingestellt. Ich wollte Ihnen damit nur demonstrieren, dass es außerordentlich schwierig ist, Nietzsche auf bestimmte Bekenntnisse festzulegen. Wenn wir diese scheinbaren Bekenntnisse nehmen und genauer hinschauen, dann tut sich ein Abgrund auf; wir sind plötzlich gar nicht mehr sicher, was er uns hier eigentlich sagen will und wozu er sich bekennt und was er von seinen Leserinnen und Lesern als Bekenntnis verlangt. Sollen wir jetzt die Jüngerinnen und Jünger des letzten Jüngers werden-Judas-Jünger? Ich weiß es nicht, und ich möchte Sie einfach einladen, bei der Nietzsche-Interpretation nicht allzu vorschnell zu verfahren. 
Abstract of the second part: It is at the very least possible to construe Nietzsche as a philosopher of immanence, because he is so clearly critical regarding the possibility of transferring the heavy weight of existence into another sphere, a transcendent sphere. This Schwergewichtsverlagerung is the main issue Nietzsche addresses in his criticism of Christianity and of asceticism. One could thus translate Nietzsche's philosophy of life into the concept of immanence. However, examining the texts more closely, we see that there is not at all a clear and definitive version of Nietzsche's philosophy of immanence, philosophy of life or philosophy of the will to power. These concepts, life and will to power-so prominent in Nietzsche-are more intellectual experiments than they are doctrines. In essence, Nietzsche is not a teacher of doctrines; rather, he is a great experimenter. And this is also the case with Dionysus. Nietzsche seems to admire and pray to the god Dionysus, but we see that Nietzsche construes himself as the last disciple of the philosopher Dionysus; and as aforementioned, the sentence "I am the last disciple of the philosopher Dionysus" seems much less clear than superficial, because Nietzsche here is clearly not interested in the god Dionysus but rather in the philosopher Dionysus. The narrator ego speaking in Twilight of the Idols is, as the last disciple, obviously a kind of Judas Iscariot-the traitor, the one who has betrayed Jesus. The narrating ego confessing his attachment/belonging to the philosopher Dionysus may thus also be a kind of traitor. Upon closer examination, the sentence quoted above is at the least not as clear as it seems to be.

Damit komme ich zum dritten und letzten Punkt und beziehe mich dabei auf Jenseits von Gut und Böse, Abschnitt 6: „Umgekehrt ist an dem Philosophen ganz und gar nichts Unpersönliches; und insbesondere giebt seine Moral ein entschiedenes und entscheidendes Zeugniss dafür ab, wer er ist - das heisst, in welcher Rangordnung die innersten Triebe seiner Natur zu einander gestellt sind." Jenseits von Gut und Böse wurde 1886 publiziert.

Wenn man ein wenig gräbt, stellt man fest, dass dieser Abschnitt eine ausgesprochen interessante Vorgeschichte hat. Eine ausgesprochen interessante Vorgeschichte, die zurückreicht zur Begegnung mit Lou von Salomé 1882 in Tautenburg, wo Nietzsche und Salomé eine intellektuelle Sommerfrische genossen, die leider von Salomés Seite außer in ihrem Tautenburger Brieftagebuch für Paul Rée (Andreas-Salomé 1970, 181-190) nicht mit zeitgenössischen Aufzeichnungen dokumentiert ist. Von Nietzsches Seite schon; da gibt es Texte aus diesem Sommer 82, die für Lou von Salomé geschrieben worden sind. (Sie sehen, dass ich sehr zögere, einfach „Lou“ zu sagen. Ich finde es merkwürdig, wenn die Nietzsche-Forscher bei Lou von Salomé von „Lou“ reden, während man, wenn von Nietzsches Freunden Franz Overbeck oder Erwin Rohde die Rede ist, nie einfach „Franz" oder „Erwin“ sagt. Also sollte man konsequent sein und entweder sich den Sprachgebrauch angewöhnen: wir haben hier Fritzens Philosophie, Franzens Theologiekritik und Lous Romanoder wir reden von Nietzsches Philosophie, Overbecks Theologiekritik und Salomés oder AndreasSalomés Roman).

In einem Brief von Nietzsche an Salomé, vermutlich vom 16.09.1882, liest man: „Meine liebe Lou, Ihr Gedanke einer Reduktion der philosophischen Systeme auf Personal-Acten ihrer Urheber ist 
recht ein Gedanke aus dem ,Geschwistergehirn': ich selber habe in Basel in diesem Sinne Geschichte der alten Philosophie erzählt und sagte gern meinen Zuhörern: ,dies Systemist widerlegt und todt - aber die Person dahinter ist unwiderlegbar, die Person ist gar nicht todt zu machen' - zum Beispiel Plato." (BVN-1882, 305) Demnach hat Nietzsche einen Brief von Salomé bekommen (dass wir diesen Brief nicht kennen, ist außerordentlich bedauerlich), in dem sie vorschlägt, die Philosophien, die philosophischen Systeme, um genauer zu sein, als Personalakten ihrer Urheber zu verstehen, also als spezifischen Ausdruck ihrer Persönlichkeit. Systeme wären demnach nichts, was die Philosophen quasi als Instrumente der Wahrheit einfach zu Papier gebracht haben ohne persönliche Beteiligung. Entsprechend ist die Philosophie Heraklits fundamental unterschieden von der Philosophie Hegels, oder die Philosophie Kants fundamental unterschieden von der Philosophie Platons. Lou von Salomé hat also ein Konzept von Philosophiegeschichte entworfen, das ich salopp "Personalaktenmethode“ nennen würdeReduktion der philosophischen Systeme auf Personalakten ihrer Urheber, so Nietzsches Formulierung. Wie gesagt, wir kennen die ursprüngliche Formulierung von Lou von Salomé nicht. Was wir hingegen haben, ist ihr Buch über Nietzsche von 1894, im Übrigen eines der besten Nietzsche-Bücher, die es überhaupt gibt. Darin druckt sie zu Beginn diesen Brief Nietzsches ab, als Faksimile, und begründet ihre eigene Methode an Nietzsche heranzugehen quasi als Versuch, Nietzsches intellektuelle Personalakten zu präsentieren. Sie nimmt die Methode, die sie mit Nietzsche 1882 entworfen hat (im Gespräch, wie auch immer) zwölf Jahre später-Nietzsche ist längst im Wahnsinn versunken-als Handreichung, um ihr eigenes Buch über Nietzsche zu schreiben.

Wir können dann, wenn wir in Nietzsches Nachlass der Sache weiter nachgehen, eine Verschiebung beobachten. Eine Verschiebung hin zu "Selbstbekenntnissen ihrer Urheber"-Sie hören da natürlich Augustinus im Hintergrund. Philosophie nicht mehr als Personalakten, das klingt zu technisch, zu bürokratisch, sondern Selbstbekenntnisse, und-das ist Nachlass 1883-nicht ein Erkenntnistrieb stehe dahinter, sondern vielmehr "Triebe, welche sich der Erkenntniß wie eines Werkzeugs bedienen“ (M III 4, 90f. bzw. KSA 14, 348f), so die Formulierung. Unversehens wird der Triebbegriff da eingetragen, der im Brief an Lou von Salomé 1882 noch keine Rolle spielte, da war nur von Personalakten die Rede. Ich erlasse Ihnen jetzt die verschiedenen Varianten, ich erlasse es Ihnen auch, nochmal auf diese von Nietzsche im Brief an Lou von Salomé erwähnte Vorlesung über die griechische Philosophie, die er in Basel gehalten hatte, zurückzugehen. Es ist in der Tat so, dass Nietzsche bereits Ähnliches versucht hatte in seiner Basler Zeit von 1869 bis 1879. Nietzsche weitete die Überlegungen dann auch aus, er sprach im Nachlass 1883 nicht mehr nur von den Philosophen, bei denen sich das in der erwähnten Weise verhalte, sondern auch von den Musikern: Erzeugnisse von Musikern seien der selben Selbstbekenntnis- und Trieblogik unterworfen wie die Erzeugnisse der Philosophen. „Der Philosoph ist nur eine Art Gelegenheit und Ermöglichung dafür, daß der Trieb einmal zum Reden kommt." (NF 1883, 7[62])

Sie sehen eine Verlagerung, eine Verlagerung hin vom Selbstbekenntnishaften, vom Personalaktenhaften, auf das Triebleben-das unbewusste Triebleben. Diese Veränderung hat mit bestimmten Lektüren Nietzsches zu tun (auch da jetzt keine Details). Nietzsche stilisiert sich oft als Autor, der völlig abgelöst ist von den zeitgenössischen Diskursen; er tut gerne so, als stünde er nur 
für sich. Steht er natürlich überhaupt nicht, vielmehr ist er begierig darauf, alles in sich aufzunehmen, was es an zeitgenössischen Debatten gibt. Wir unterliegen einer optischen Täuschung, wenn wir denken, Nietzsche sei, wie er sich selber gerne stilisiert, auf einem Denkergipfel, unberührt von allem, außer den ganz Großen, die es vielleicht in der Vergangenheit noch gegeben hat. Man sieht dann Nietzsche auf dem Gipfel quasi winken, zurück zu Spinoza, zurück zu Platon. Faktisch ist es jedoch so, dass er sich in ständiger Auseinandersetzung mit der zeitgenössischen Literatur bewegt. Und wir unterliegen einer optischen Täuschung, weil wir diese Literatur normalerweise gar nicht mehr kennen. Nietzsche ist ein hochgradig reaktiver Denker; er nimmt immer die Anregungen des Tages auf; und je nachdem, was er gerade für Bücher unter seinem Kopfkissen, in seiner Hand hat, fallen die Reaktionen auch anders aus. Aber wie gesagt, auf die Details zum Triebleben und zur Triebliteratur muss ich hier aus Platzgründen verzichten.

Interessant ist die Veränderung des Begriffs von Philosophiegeschichte, den wir an dieser Stelle beobachten können. „Die einzige Kritik einer Philosophie“-so heißt es früher, 1874, in der Dritten unzeitgemäßen Betrachtung über Schopenhauer als Erzieher, 8. Abschnitt -, "die möglich ist und die auch etwas beweist, nämlich zu versuchen, ob man nach ihr leben könne, ist nie auf Universitäten gelehrt worden: sondern immer die Kritik der Worte über Worte." (SE-8) Das ist eine ganz klassische Kritik an der Philosophie, die Nietzsche beispielsweise auch bei Schopenhauer oder Emerson hatte finden können. Der Referenzbegriff ist das Leben-das klingt so, als würde das analog gehen mit all dem, was wir zu den Trieben gehört haben, geht aber nicht wirklich analog. Interessant ist, dass die Triebe keine Rolle spielen in diesem frühen Konzept von Philosophie. Bei näherem Hinsehen zeigt sich vielmehr, dass auch der Lebensbegriff noch anders bestimmt ist. Er hat diese Triebcharakteristik noch nicht derart eingeschrieben bekommen, wie es sich dann in den 80er Jahren vor dem Hintergrund naturwissenschaftlicher Lektüren vollzieht. Es geht in Abschnitt 6 von Jenseits von Gut und Böse nicht mehr darum, eine Philosophie zu propagieren, die das Leben bestimmen soll. Das war noch die Exposition in Schopenhauer als Erzieher 1874, derzufolge wir eine Philosophie brauchen, die das Leben bestimmt. In Jenseits von Gut und Böse 1886 ist das Verhältnis umgekehrt. Jetzt ist es so, dass das Leben durch die Triebe die Philosophie bestimmt. Mit anderen Worten: Wir haben den scheinbar selben Leitterminus, nämlich „Leben“, aber die Verhältnisbestimmung ist genau umgekehrt. Jetzt ist es so, dass sich das Leben unter anderem in Philosophie ausdrückt. Nicht länger benötigen wir eine Philosophie, mit der wir das Leben bewältigen können, sondern das Leben bewältigt sich in der Philosophie immer schon selber. Und je nachdem, welche Art von Leben, welche Lebensform wir haben, haben wir auch andere Formen von Philosophie.

Der Trieb zur Erkenntnis ist offensichtlich nichts, was in einem selbstzweckhaften Sinne zu verstehen wäre. Erkenntnistrieb ist ein Trieb, der sich der Erkenntnis nur als eines Werkzeuges bedient, so heißt es weiter im Abschnitt 6 von Jenseits von Gut und Böse. Die Gelehrten, wird dort ausgeführt, seien vielleicht diejenigen, die einen Erkenntnistrieb haben, weil sie nicht wirklich von Leidenschaft bewegt sind. "Umgekehrt ist an dem Philosophen ganz und gar nichts Unpersönlichesein entschiedenes und entscheidendes Zeugniss dafür ab, wer er ist" (JGB-6). In der Diskussion des 19. Jahrhundert gilt Unpersönlichkeit oft als Inbegriff der Moral. Menschen sollen in Ihrem moralischen Handeln eben nicht persönlich involviert sein, das scheint von Kant her 
evident: Kein persönliches Involviertsein, keine Parteinahme fürs Eigene. Das hält Nietzsche in ethicis für eine höchst problematische Perspektivierung. Menschliches, Allzumenschliches I, Abschnitt 95 von 1878 gab dafür schon die Leitlinie vor. Abschnitt 6 von Jenseits von Gut und Böse bezieht sich darauf. Für Nietzsche ist es eine Illusion, dass man das Persönliche ausschalten könne, im Hinblick auf Moral im weitesten Sinne. Es ist aber auch eine Illusion in Hinblick auf die Ästhetik. Nietzsche hat sich in den Briefen von Gustave Flaubert an George Sand eine Passage am Rand markiert: „Je crois que le grand art est scientifique et impersonnel." (Flaubert 1884, 41) Flaubert glaubt also, dass die große Kunst wissenschaftlich und unpersönlich sei. Und das ist die genaue Gegenposition zu Nietzsches eigenem Verständnis von Kunst und Philosophie. Es ist sozusagen sein Versuch, sich gegen den Szientismus seiner Zeit zu behaupten.

Ich komme damit zur letzten Textstelle. Es geht um Jenseits von Gut und Böse, Abschnitt 3. As I mentioned at the beginning, I have some difficulties in that the nuances are improperly expressed in the English Nietzsche discussion and particularly in the Nietzsche translations. From my point of view, the translation offered in the conference reader ${ }^{2}$ is in many respects, to put it politely, rather misleading. To simply begin at the beginning: In the German text, you have a contrast drawn between fingers and eyes ("Nachdem ich lange genug den Philosophen zwischen die Zeilen und auf die Finger gesehn habe" (JGB-3)). Those fingers, however, suddenly disappear in the English text: "I have kept a close eye on the philosophers and read between their lines for long enough to say myself [...]" (Nietzsche 2002, 6-7)—Where are the fingers? Then, continuing: "Man muss hier umlernen." The English text renders umlernen as "re-examination" (Nietzsche 2002, 7 bzw. JGB-3). "Relearn," "trans-learn" or something of the like would offer us a more adequate translation; "reexamination" only means that you, well, look again, while umlernen means that you have changed your mind. Or take the very last sentence of the English translation: "But this assumes that it is not man who is the 'measure of things"' (Nietzsche 2002, 7). The German text does not have this idea of consequence; it does not follow from what has been said before. Gesetzt nämlich is not at all an indication that we must induce a consequence from what precedes but is rather an elliptical form of speaking. This elliptical form of speaking has totally disappeared in the English translation. One should perhaps translate it with "Given that" or "Let us assume that;" but then we would also have a complete sentence whereas, in the German original, we do not have a grammatically complete sentence. And the gerade - "even"-has also disappeared in the English text. Hence, this translation of Beyond Good and Evil should really be reworked to provide a more adequate sense of the meaning of the text.

Zurück zum deutschen Text. Also, Übersetzungen Nietzsches sind ausgesprochen schwierig, und ich glaube, man wäre oft besser beraten, den Texten eine zeilengenaue Übersetzung angedeihen zu lassen, als eine interpretierende, wie üblich. Natürlich ist es auch auf Deutsch irritierend, dass man nicht nur zwischen den Zeilen lesen, sondern auch auf die Finger schauen soll. Aber man müsste, wenn man ernsthaft übersetzen will, diese Nuance zu übertragen versuchen, gerade damit es für fremdsprachige Leser genauso irritierend wirkt wie für deutschsprachige Leser_innen. 
„[M]an muss noch den grössten Theil des bewussten Denkens unter die Instinkt-Thätigkeiten rechnen, und sogar im Falle des philosophischen Denkens." (JGB-3) Dieser Fokus scheint uns mit Nietzsche völlig selbstverständlich. Ja, Instinkte, davon hat er doch immer geredet. Aber: Ganz so einfach ist das nicht. Wir begegnen dem Instinktbegriff zwar schon beim ganz frühen Nietzsche. $\mathrm{Er}$ benutzte ihn da in denunziatorischer Absicht—etwa gegen Euripides. Euripides nämlich, der griechische Tragödiendichter, bekommt als Vorwurf serviert, er habe die Instinkte, das Instinktive verraten. Bei den Griechen soll, so Nietzsche 1872, offensichtlich so etwas wie eine Instinktharmonie bestanden haben. Und Euripides, zusammen mit Sokrates, sei derjenige gewesen, der das Ganze destruktiv umgepflügt habe.

Schon beim frühen Nietzsche gibt es einen Instinktbegriff. Man könnte denken, das sei alles irgendwie dasselbe, ist es aber nicht. Nietzsche reichert den Instinktbegriff, den er zunächst einmal der Kulturpublizistik seiner Zeit entnimmt, nach und nach naturwissenschaftlich an. Eine wichtige Quelle ist dafür Georg Heinrich Schneiders Der menschliche Wille vom Standpunkte der neueren Entwicklungstheorien von 1882-Sie sehen am Titel schon, dass es hier um Evolutionsbiologie geht. Und die Evolutionsbiologie ist eine jener Disziplinen, die Nietzsche um der naturwissenschaftlichen Aufrüstung seiner Philosophie willen in den 1880er Jahren in sein Denken einbezieht. Schneiders Buch ist in Nietzsches Bibliothek erhalten. Da gibt es eine Passage, die lautet: „Das instinctive Handeln bildet [...] in jeder Hinsicht die Grundlage zu den Willensäusserungen im engeren Sinn. [...] Instinct ist das psychische Streben nach Arterhaltung ohne Bewusstsein des Zweckes von diesem Streben." (Schneider 1882, 109) Instinkte sind demnach unbewusst; sie dienen der Arterhaltung, so die darwinistische Konzeption bei Schneider. Eine darwinistische Konzeption, die Nietzsche jedoch nicht einfach übernimmt, sondern die er einpasst in sein eigenes Denken. Er zeigt sich gegenüber Darwin sehr kritisch, aber das ist wieder ein anderes Thema (vgl. Sommer 2010). Wesentlich ist an dieser Stelle, dass er mit Darwin den Menschen als historisch, als evolutionär-historisch gewordenes Wesen denkt. Als Wesen, das geworden ist im Laufe einer Entwicklungsgeschichte. Dass er in den Details von Darwin und von den Darwinisten stark abweicht, steht außer Zweifel. Aber die Grundintention, zu sagen, der Mensch sei nicht einfach, sondern er sei ein gewordenes Wesen, ein biologisch gewordenes Wesen, das ist für Nietzsche von Darwin her eine unhintergehbare Voraussetzung. Ein zweiter Autor, den Nietzsche benutzt, um das Instinktive neu zu justieren, ist Otto Liebmann, ein wichtiger neukantianischer Philosoph, der aber auch in der Naturwissenschaft zuhause ist. Ein Aufsatz "Ueber den Instinct", 1880 erschienen, wurde von Nietzsche mit vielen Glossen, Anstreichungen und positiven Äußerungen am Rand versehen. Nietzsche schreibt in seinen Büchern sehr häufig unzimperliche Urteile hin, nicht allzu selten „Esel!“ oder „Idiot!“. Hier bei Liebmann steht hingegen bei einer Passage wie der Folgenden-Nietzsches Unterstreichungen—am Seitenrand ein emphatisches ,ja!":

Andererseits sind die bewunderungswürdigsten Functionen des menschlichen Geistes, sittlicher Tact und künstlerisches Genie, mit dem Instinct der Thiere wesentlich einerlei, nur graduell von inm verschieden mit ihm offenbar unter dasselbe Genus gehörig, nämlich, wie er anscheinend Ausflüsse einer höheren Inspiration. Instinct, Tact, Geniekommen darin überein, daß sie unüberlegt das Richtige und Angemessene fühlen, wollen und thun; sie bestehen in der Fähigkeit, ohne Vorbedacht, ohne Erfahrung und Einübung, ohne Reflexion und Prämeditation, 
Also, die Bruchstücke zu einer Theorie des Instinktes bei Nietzsche sind in zeitgenössische Diskussionen eingebunden und kombinieren biologische Bedingtheit mit historischer und sozialer Bedingtheit. Instinkt ist nichts, was uns einfach von einem biologischen Programm auferlegt wird. Sondern Instinkt ist etwas, was entsteht, was gemacht ist. Aber nicht von uns persönlich gemacht, sondern, was wir ererben, ererben von den Eltern, den Ureltern, aber auch von den Einzellern, die in unserer Stammesgeschichte am Anfang standen.

"Gesetzt nämlich, dass nicht gerade der Mensch das ,Maass der Dinge' ist..."-ich sagte schon, dass ich Judith Normans englische Übersetzung dieses letzten Satzes in Jenseits von Gut und Böse, Abschnitt 3 für irreführend halte, weil sie suggeriert, dass hier eine Folgerung gezogen werde aus dem Vorangegangenen, was gerade nicht der Fall ist. Evident ist der philosophiehistorische Bezug, nämlich auf den homo-mensura-Satz des Protagoras von Abdera, demzufolge "der Mensch das Maß aller Dinge" sei, „der seienden, daß sie sind, der nicht seienden, daß sie nicht sind“. Bemerkenswert ist die Öffnung hin zum Unbestimmten, während davor im selben Abschnitt 3 von Jenseits von Gut und Böse der Irrglaube der Philosophien kritisiert worden ist, "dass das Bestimmte mehr werth sei als das Unbestimmte“. Der Satz am Ende gibt keine bestimmte Alternative, wie man erwarten würde-etwa die bestimmte Aussage, nicht der Mensch sei das Maß aller Dinge, sondern der Instinkt, oder das Tier im Menschen oder der Einzeller im Menschen oder was auch immer. Nein, das macht Nietzsche nicht. Wir haben hier eine Setzung, eine hypothetische Setzung, dass der Mensch nicht das Maß aller Dinge sei, aber der Text verweigert die Auskunft, was stattdessen das Maß aller Dinge ist. Es wird keine Alternative geboten. Wir können mutmaßen, Nietzsche meine vielleicht, dass nicht die Vernunft das Maß aller Dinge sei, wie die Philosophen gerne glaubten. Dass es keinen abstrakten Menschen gebe, der das Maß aller Dinge sei. Im Ausgangstext des

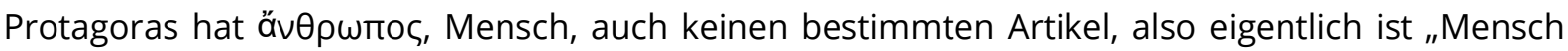
Maß aller Dinge"—es fragt sich, ob das generalisierend gemeint ist oder individualisierend. Bei allen Mutmaßungen entscheidend scheint mir die Öffnung hin auf eine philosophische Denkanstrengung zu sein, die die Leserin, der Leser selbst vollziehen muss. Wir bekommen im Text keine Antwort darauf, was jetzt das Maß aller Dinge ist. Ich will nur in Klammern bemerken, dass wir hier einen intertextuellen Bezug zu Menschliches, Allzumenschliches I, Abschnitt 2 haben, wo davon gehandelt wird, dass den Philosophen ",der Mensch“ als eine aeterna veritas, als ein Gleichbleibendes in allem Strudel, als ein sicheres Maass der Dinge“ vorschwebe. Dieser Abschnitt problematisiert gleichfalls die Vorstellung einer feststehenden Maßstäblichkeit des Menschen und empfiehlt stattdessen ein historisches Philosophieren. Ein Philosophieren, das den Menschen als ein historisches Wesen versteht, ein Wesen, das ein gewordenes Wesen ist. 
Very brief abstract: In dealing with Sections 3 and 6 of Beyond Good and Evil, I attempt to show that what seems so clear on the surface is not at all clear when we take a closer look the text. We must consider the background, the context into and from which Nietzsche wrote. Thus, in our interpretation of Nietzsche, we must avoid propagandizing the big terms, believing that we can pin him down just with those big terms. We must read and interpret Nietzsche carefully because we are always involved in intellectual experiments; Nietzsche performs intellectual experiments not only with his texts but also with his readers-with us as his readers.

Ganz zum Schluss, Sie sehen meine Damen und Herren, als Resümee, dass es keineswegs klar ist, was bei Nietzsche das Maß aller Dinge ist. Offenbar sind es keine immanenten Gesetzmäßigkeiten (womit ich wieder ganz zum Anfang komme), die es irgendwie gibt da draußen, denen wir uns einfach fügen müssten, wie vielleicht die Vorsokratiker und viele Philosophen nach ihnen geglaubt haben. Es sind offensichtlich sehr individuelle und historisch-biologisch-sozial gewordene Maßstäbe, die Gesetze sind, aber nur individuelle Gesetze, je nachdem, als was wir uns ausprägen. Es ist nicht so, dass Leben einen Einheitsausdruck hätte, sondern Leben bedeutet ganz offensichtlich im höchstem Maße Pluralität. Zur Frage, die ich im Titel dieser Überlegungen gestellt habe, ob Nietzsche ein Immanentist ist, sehen Sie natürlich meine skeptischen Bedenken. Ich würde Nietzsche überhaupt auf kein -ist-Sein und auf keinen -ismus festlegen wollen. Nietzsche ist nicht "der Willensphilosoph" oder "der Lebensphilosoph" oder "der amor-fati-Philosoph"; er ist alles Mögliche und alles zugleich und alles zugleich auch nicht. Also er ist ebenso wenig Immanentist, wie er es nicht ist, wenn Sie mir diese paradoxe Formulierung erlauben.

Vielleicht hängt es auch nicht an privilegierten Orten, an denen Immanenz in Erscheinung tritt. Vielleicht ist überall und immer die Fülle des Daseins, die Eigentlichkeit gegeben. Vielleicht haben wir keine besonderen Orte, wo wir das Eigentliche, die Authentizität zum Beispiel erleben könnten, und viele andere Orte, wo wir das nicht können. Warum annehmen, es gebe eine Eigentlichkeit, die irgendwo verborgen liegt, statt die Eigentlichkeit hier und jetzt und in jedem Augenblick realisiert zu finden? Ich gebe gerne zu, dass diese allerletzte Schlussfolgerung eine ist, die ich selber ziehen würde und bei der Nietzsche womöglich, und ich hoffe es auch, größte Bedenken und Einreden formulieren würde.

\section{Endnoten}

\footnotetext{
${ }^{1}$ Alle Nietzsche-Originalzitate dieses Beitrages folgen der von Paolo D'lorio herausgegebenen Digitalen kritischen Gesamtausgabe nach Giorgio Colli und Mazzino Montinari unter http://nietzschesource.org/\#eKGWB.

2 Die Übersetzung im Konferenz-Reader stammt aus: Nietzsche (2002).
} 


\section{Bibliographie}

Andreas-Salomé, Lou. 1970. „Tagebuch für Paul Rée.“" In Die Dokumente ihrer Begegnung, auf der Grundlage der einstigen Zusammenarbeit mit Karl Schlechta und Erhart Thierbach herausgegeben von Ernst Pfeiffer, 181-190. Frankfurt/Main: Insel Verlag.

Flaubert, Gustave. 1884. Lettres à George Sand. Précédées d'une étude par Guy de Maupassant. Paris: C. Charpentier.

Liebmann, Otto. 1880. Zur Analysis der Wirklichkeit. Eine Erörterung der Grundprobleme der Philosophie. Zweite, beträchtlich vermehrte Auflage. Straßburg: Trübner.

Nietzsche, Friedrich. 1999. Sämtliche Werke. Kritische Studienausgabe in 15 Einzelbänden. Herausgegeben von Giorgio Colli und Mazzino Montinari. 3. Auflage. München/Berlin/New York: DTV Walter de Gruyter [=KSA].

2002. Beyond Good and Evil. Prelude to a Philosophy of the Future. Übersetzt von Judith Norman. Cambridge: Cambridge University Press.

Schneider, Georg Heinrich. 1882. Der menschliche Wille vom Standpunkte der neueren Entwicklungstheorien (des „Darwinismus"). Berlin: Ferd. Dümmlers Verlagsbuchhandlung.

Sommer, Andreas Urs. 2010. „Nietzsche mit und gegen Darwin in den Schriften von 1888." Nietzscheforschung. Jahrbuch der Nietzsche-Gesellschaft 17: 31-44.

2012. Nietzsche-Kommentar: Der Fall Wagner und Götzen-Dämmerung. Historischer und kritischer Kommentar zu Friedrich Nietzsches Werken. Bd. 6/1. Berlin/Boston: Walter de Gruyter.

\section{Biographie}

Professur für Philosophie mit Schwerpunkt Kulturphilosophie / Akademie-Professur in Kooperation mit der Heidelberger Akademie der Wissenschaften.

Geboren 1972, Studium der Philosophie, Kirchen- und Dogmengeschichte und Deutschen Literaturwissenschaft in Basel, Göttingen und Freiburg im Breisgau, Lizentiat 1995, Promotion 1998 an der Universität Basel, 1998/99 Visiting Research Fellow an der Princeton University, 2000-2006 Wissenschaftlicher Assistent am Institut für Philosophie der Universität Greifswald, 2001 Visiting Fellow an der School for Advanced Study der University of London, Habilitation 2004 an der Universität Greifswald, 2007 Lehrstuhlvertretung an der Universität Mannheim, 2008-2014 Wissenschaftlicher Kommentator der Werke Nietzsches, Direktor der Friedrich-Nietzsche-Stiftung, 2013/14 Gastprofessur an der Staatlichen Hochschule für Gestaltung in Karlsruhe, seit 2014 Leiter der Forschungsstelle "Nietzsche-Kommentar" der Heidelberger Akademie der Wissenschaften, 2011-2016 außerplanmäßiger, seit 2016 ordentlicher Professor für Philosophie mit Schwerpunkt Kulturphilosophie an der der Universität Freiburg.

(c) 2017 Andreas Urs Sommer

(1)

Dieses Werk ist, sofern nicht anders angegeben, lizenziert unter einer Creative Commons

Namensnennung - Nicht-kommerziell - Weitergabe unter gleichen Bedingungen 4.0 International Lizenz. 